\title{
Topic specific association rules mining from social bookmarks
}

\author{
Ilija Subasic \\ Faculty of Economics Subotica, Segedinski put 9-11, \\ 24000 Subotica, Serbia, www.eccf.su.ac.yu \\ ilija@eccf.su.ac.yu
}

\begin{abstract}
In recent years social bookmarking systems have become one of the most popular application on the Internet enabling users to label some content. The user's perspective of this content is expressed with some words (tags). The main goal of this paper is to propose a method for finding association between tags and topic, as well as the association among the tags for a specific topic. Due to the difference in user opinions in the meaning of a word, the same word can be used to tag some content but within a different context. Application of association rules to tagged content neglects two things, the content itself and context of the rule. To overcome this, two new metrics are introduced to a standard apriori algorithm. Relevance support and relevance confidence are calculated as the association of a tag to a topic or an another tag with a respect to the relevance of a tagged content to a specific topic. This can be used as a input of a recommender system by recommending items depending on a topic that the user is requesting, or it can be used for navigation of a crawler that collects web pages from a social bookmark site.
\end{abstract}

Keywords: association rules, relevant confidence and relevant support, social bookmarking, folksonomies 